EPJ manuscript No.

(will be inserted by the editor)

\title{
The study of the negative pion production in neutron-proton collisions at beam momenta below $1.8 \mathrm{GeV} / \mathrm{c}$
}

\author{
V.V. Sarantsev ${ }^{1}$, K.N. Ermakov ${ }^{1}$, L.M. Kochenda ${ }^{1}$, V.I. Medvedev ${ }^{1}$, V.A. Nikonov ${ }^{1,2}$, O.V. Rogachevsky ${ }^{1}$, \\ A.V. Sarantsev ${ }^{1,2}$, S.G. Sherman ${ }^{1}$, V.A. Trofimov ${ }^{1}$, and A.A. Vasiliev ${ }^{1}$ \\ 1 Petersburg Nuclear Physics Institute, Gatchina 188300, Russia \\ 2 Helmholtz-Institut für Strahlen- und Kernphysik, Universität Bonn, Germany
}

\begin{abstract}
A detailed investigation of the reaction $n p \rightarrow p p \pi^{-}$has been carried out using the data obtained with the continuous neutron beam produced by charge exchange scattering of protons off a deuterium target. A partial wave event-by-event based maximum likelihood analysis was applied to determine contributions of different partial waves to the pion production process. The combined analysis of the $n p \rightarrow p p \pi^{-}$ and $p p \rightarrow p p \pi^{0}$ data measured in the same energy region allows us to determine the contribution of isoscalar partial waves $(I=0)$ in the momentum range from 1.1 up to $1.8 \mathrm{GeV} / \mathrm{c}$. The decay of isoscalar partial waves into $\left({ }^{1} S_{0}\right)_{p p} \pi$ channel provides a good tool for a determination of the $p p$ S-wave scalar scattering length in the final state which was found to be $a_{p p}=-7.5 \pm 0.3 \mathrm{fm}$.
\end{abstract}

PACS. 13.75.Cs Nucleon-nucleon interactions - 13.85.Lg Total cross sections - 25.40.Fq Inelastic neutron scattering

\section{Introduction}

The single pion production in $N N$ collisions is the main inelastic process at energies below $1 \mathrm{GeV}$. Despite the fact that a lot of experiments have been performed in this region, many questions on this process are still far from being satisfactory answered. One of them is the question about contributions of isoscalar $(I=0)$ partial waves to the inelastic neutron-proton collision. The neutron-proton scattering amplitude contains both isoscalar and isovector $(I=0,1)$ parts, and while the isovector part is rather well known, even the magnitude of the total isoscalar crosssection is badly determined. Usually, this cross section is extracted from the difference of the total cross-sections of the pion production reactions: $n p \rightarrow p p \pi^{-}$and $p p \rightarrow$ $p p \pi^{\circ}$. However the numbers obtained in different experiments are fairly scattered and do not give the whole picture for the behavior of the isoscalar cross-section in the energy region below $1 \mathrm{GeV}$. The only conclusion which can be made from these values is that the contribution of the isoscalar cross section to the $n p \rightarrow p p \pi^{-}$reaction is smaller by about one order of magnitude than the contribution of the isovector one.

It should be noted that the experimental data on the $n p \rightarrow p p \pi^{-}$reaction below the energy of $1 \mathrm{Gev}$ are much poorer than those for $p p$ collisions. The main reason for this situation is due to the difficulty to create a pure monoenergetic neutron beam. Therefore most previous experiments used either a continuous neutron beam in a fairly wide energy range [1] or investigated the $p n$-interaction with a proton beam and a deuteron target as a neutron source [2,3,4. Of course, it is worth to mention the works where the energies of neutrons were defined using the timeof-flight technique [5,6].

In the present study we use the continuous neutron beam produced by the $p d$ interactions on the deuterium target. For a determination of the contributions of the isoscalar cross-section we performed a partial wave analysis of the data in whole region of incident neutron momenta combined with the $p p \rightarrow p p \pi^{0}$ data. In such an approach, the contribution of the isoscalar channel can be determined, for example, from the asymmetry of the pion distribution in the c.m.s. of the reaction, which is defined by the interference of isoscalar and isovector amplitudes. Thus the small isoscalar amplitudes can be defined with good accuracy.

\section{Experimental set up and data selection}

The $n p \rightarrow p p \pi^{-}$data were taken at the PNPI synchrocyclotron with the $35 \mathrm{~cm}$ hydrogen bubble chamber disposed in the $1.48 \mathrm{~T}$ magnetic field. The neutrons were produced from collision of $1 \mathrm{GeV}$ energy proton beam with thinwalled liquid deuterium target. The charged particles are swept away from the created neutron beam by a system of the additional magnets and lenses situated behind the deuteron target. The neutron beam passed through a collimator with size $100 \times 2 \times 1 \mathrm{~cm}^{3}$ and then the distance of $5 \mathrm{~m}$ to the bubble chamber. The produced neutrons had a 
momentum spread in a range around $1 \div 2 \mathrm{GeV} / \mathrm{c}$. The detailed description of the neutron beam and its energy distribution can be found in 7 .

A total of $10^{5}$ stereofilms were obtained with this beam. The films were scanned twice with a goal to select events with two positive curvature tracks and one negative track. Due to small density of tracks on frames the efficiency of such selection was near to $100 \%$. As the result, 10835 three prong events were found. Those events can belong either to the single pion production reaction

$$
n p \rightarrow p p \pi^{-}
$$

or to the double pion production reactions

$$
\begin{aligned}
& n p \rightarrow n p \pi^{+} \pi^{-}, \\
& n p \rightarrow d \pi^{+} \pi^{-} .
\end{aligned}
$$

To separate the first process from the other two, tracks of events disposed in the fiducial volume of the chamber were measured, geometrically reconstructed and the momenta of assumed particles were determined in according with their masses. The identification of the events was performed by a kinematic fit demanding that the confidence level of accepted events is better then $1 \%$. The direction of the incident neutron beam is known from the collimating system which provides the direction with the angular spread of $0.3^{\circ}[7$. But nothing is known about a value of the neutron momentum which had to be defined by a 3Cconstrained fit. Three prong events were constrained by the kinematical fits for all three mentioned above reactions (1)-(3). A visual scan of a bubble density on the tracks was carried out for events fitted by several hypotheses in order to distinguish between pion, proton and deuteron. The angular resolution of the direction of the incident neutron beam both in azimuthal and in polar angles was first estimated to the $1^{\circ}$ which turned out to be more than three times larger than that obtained after the fit. After repeated fits with corrected errors we selected 8251 events assigned to reaction (1). In the present experiment we did not monitor the neutron beam and therefore the momentum distribution is given not in milibarns but in the number of events. To determine the absolute isoscalar cross section we included in our partial wave analysis the $p p \rightarrow$ $p p \pi^{o}$ data for which the total cross section was measured in our previous experiments.

Fig. 1] shows the neutron beam distribution for selected $n p \rightarrow p p \pi^{-}$events. The neutron momentum distribution shows a peak approximately at the energy of the original protons incident on the deuterium target. The second peak with less energetic neutrons is created in inelastic processes.

Angular distributions of pions and protons in the c.m.s. of the reaction for chosen intervals of the initial neutron momenta are shown in Fig. 2. The proton angular distributions are rather symmetrical due to permutation of protons, while the pion angular distributions show a clear asymmetry (especially at low momenta of incident neutrons). While isovector amplitudes are symmetrical with respect to the direction of the produced pion, the isoscalar

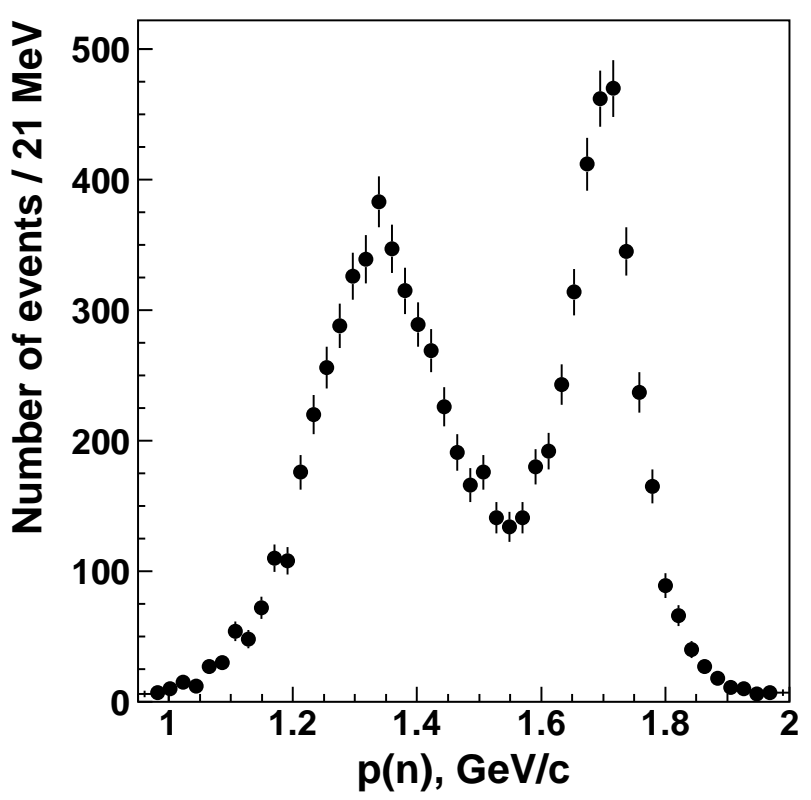

Fig. 1. The neutron beam distribution for events of the reaction $n p \rightarrow p p \pi^{-}$.

amplitudes are antisymmetrical ones. Therefore, the asymmetry in the differential cross section is defined by the interference of these amplitudes providing a sensitive tool for a determination of the weak isoscalar partial waves.

The two body invariant mass distributions of final particles are shown in Fig. 3. The $\pi p$ invariant mass at high energies of the neutrons has a peak defined by the production of $\Delta(1232)$ state and indeed, as the partial wave analysis has shown, the $\Delta(1232) p$ channel is one of dominant final states in this reaction. The $p p$ invariant mass distributions are smooth and do not reveal any peculiarities.

\section{Parameterization of partial waves}

For the production of three particles with the 4-momenta $q_{i}$ from two particles colliding with 4-momenta $k_{1}$ and $k_{2}$, the cross section is given by:

$$
d \sigma=\frac{(2 \pi)^{4}|A|^{2}}{4|\boldsymbol{k}| \sqrt{s}} d \Phi_{3}\left(P, q_{1}, q_{2}, q_{3}\right), \quad P=k_{1}+k_{2},
$$

where $A$ is the reaction amplitude, $\boldsymbol{k}$ is the 3 -momentum of the initial particle calculated in the c.m.s system of the reaction, and $s=P^{2}=\left(k_{1}+k_{2}\right)^{2}$. The invariant three particle phase space is given by

$$
d \Phi_{m}\left(P, q_{1} \ldots q_{m}\right)=\delta^{4}\left(P-\sum_{i=1}^{3} q_{i}\right) \prod_{i=1}^{3} \frac{d^{3} q_{i}}{(2 \pi)^{3} 2 q_{0 i}} .
$$

The total amplitude can be written as sum of the partial wave amplitudes:

$$
\begin{array}{r}
A=\sum_{\alpha} A_{t r}^{\alpha}(s) Q_{\mu_{1} \ldots \mu_{J}}^{i n}(S L J) A_{2 b}\left(i, S_{2} L_{2} J_{2}\right)\left(s_{i}\right) \times \\
Q_{\mu_{1} \ldots \mu_{J}}^{f i n}\left(i, S_{2} L_{2} J_{2} S^{\prime} L^{\prime} J\right) .
\end{array}
$$



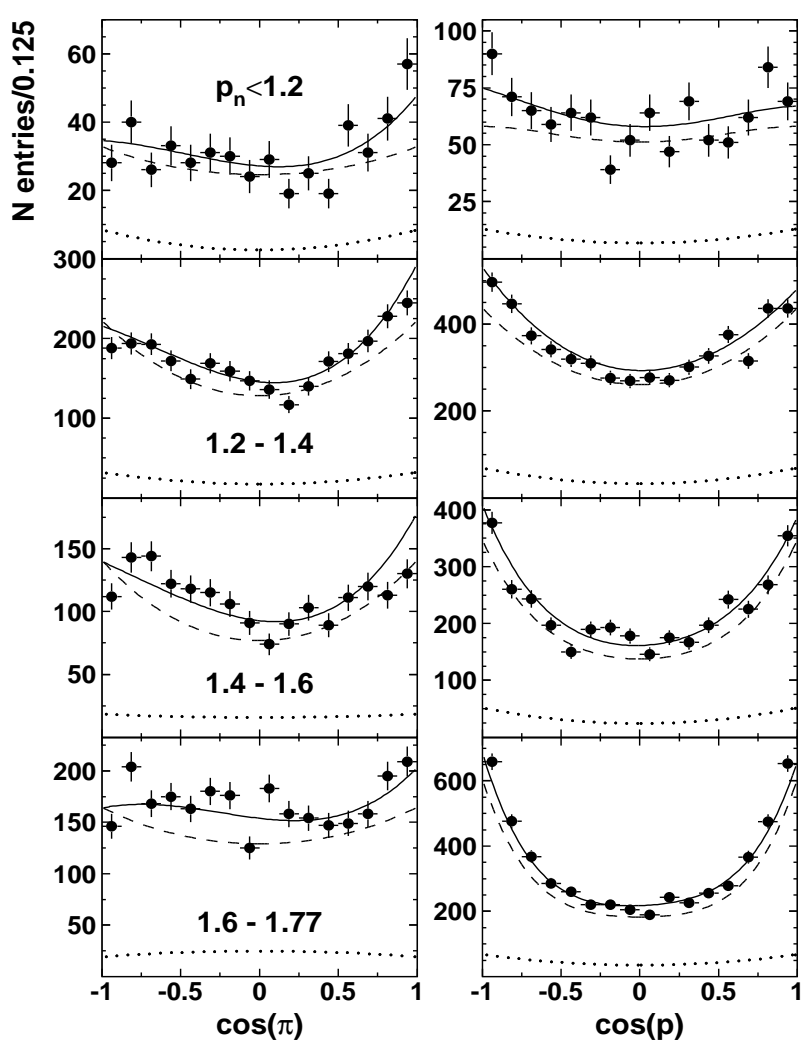

Fig. 2. The angular distribution of pion (left-hand column) and final proton (right-hand column) in the c.m.s. of the $n p \rightarrow p p \pi^{-}$reaction for 4 intervals of neutron momentum (in $\mathrm{GeV} / \mathrm{c}$ ). The full curves are the result of the partial wave analysis, the dashed curves are contributions from isovector amplitudes and dotted curves are contributions from isoscalar amplitudes.

Here $S, L, J$ are spin, orbital momentum and total angular momentum of the $n p$ system, $S_{2}, L_{2}, J_{2}$ are spin, orbital momentum and total angular momentum of a twoparticle system in the final state and $S^{\prime}, L^{\prime}$ are spin and orbital momentum between the two particle system and a third particle with momentum $q_{i}$. The invariant mass of the two body system can be calculated as $s_{i}=\left(P-q_{i}\right)^{2}$. The multiindex $\alpha$ denotes all possible combinations of the $S, L, J, S_{2}, L_{2}, J_{2}, S^{\prime}, L^{\prime}$ and $i, A_{t r}^{\alpha}(s)$ is the transition amplitude and $A_{2 b}\left(i, S_{2} L_{2} J_{2}\right)\left(s_{i}\right)$ describes rescattering processes in the final two particle channel (e.g. production of $\Delta(1232))$. In this spin-orbital momentum decomposition we follow the formalism given in $8,9,10$. The exact form of the operators for initial states $Q_{\mu_{1} \ldots \mu_{J}}^{i n}(S L J)$ and final states $Q_{\mu_{1} \ldots \mu_{J}}^{f i n}\left(i, S_{2} L_{2} J_{2} S^{\prime} L^{\prime} J\right)$ can be found in [10].

Following this decomposition we use the usual spectroscopic notation ${ }^{2 S+1} L_{J}$ for description of an initial state, the system of two final particles and the system "spectator and two-final particle state". For the initial $n p$ system, states with total momenta $J \leq 2$ and angular momenta between two nucleons of $L=0,1,2,3$ were taken into account. For the final three particle system we restricted the fit by angular momenta $L_{2}=0,1,2$ and $L^{\prime}=0,1,2$.
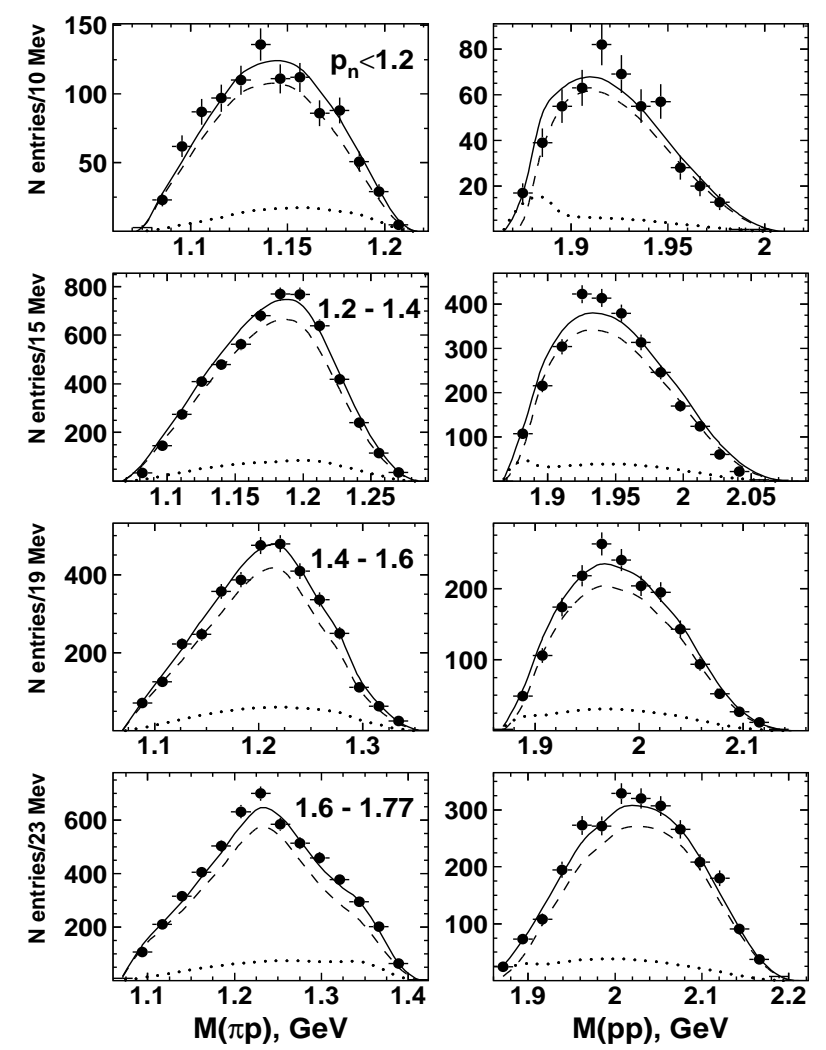

Fig. 3. The $\pi p$ and $p p$ invariant mass distributions of the final particles for 4 intervals of neutron momentum (in $\mathrm{GeV} / \mathrm{c}$ ). The full curves are the result of the partial wave analysis, the dashed curves are contributions from isovector amplitudes and dotted curves contributions from isoscalar amplitudes.

Due to the nonresonant nature of the $n p$ system in the energy region investigated here, there is no factorization between initial and final vertices and the transition amplitude can depend on all quantum numbers which characterize a partial wave (index $\alpha$ ). Moreover, due to contribution of the triangle singularities, the production parameters can be complex numbers. The best description was obtained with the parameterization

$$
A_{t r}^{\alpha}(s)=\frac{a_{1}^{\alpha}+a_{3}^{\alpha} \sqrt{s}}{s-a_{4}^{\alpha}} e^{i a_{2}^{\alpha}},
$$

where $a_{i}^{\alpha}$ are real parameters. The $a_{4}^{\alpha}$ parameter corresponds to a pole situated in the region of left-hand side singularities of the partial wave amplitudes. It is introduced to suppress the growing of the amplitudes at the regions of large $s$.

We also used other, more complicated parameterizations of the transition amplitude. However, either we obtained a worse description of the data or a similar description with larger number of parameters in the fit. In the latter case we included those results to determine the final systematical errors in the isoscalar contribution to the cross section.

For the $\pi p$ system in the intermediate state we introduce two resonances, $\Delta(1232) P_{33}$ and Roper $N(1440) P_{11}$. 

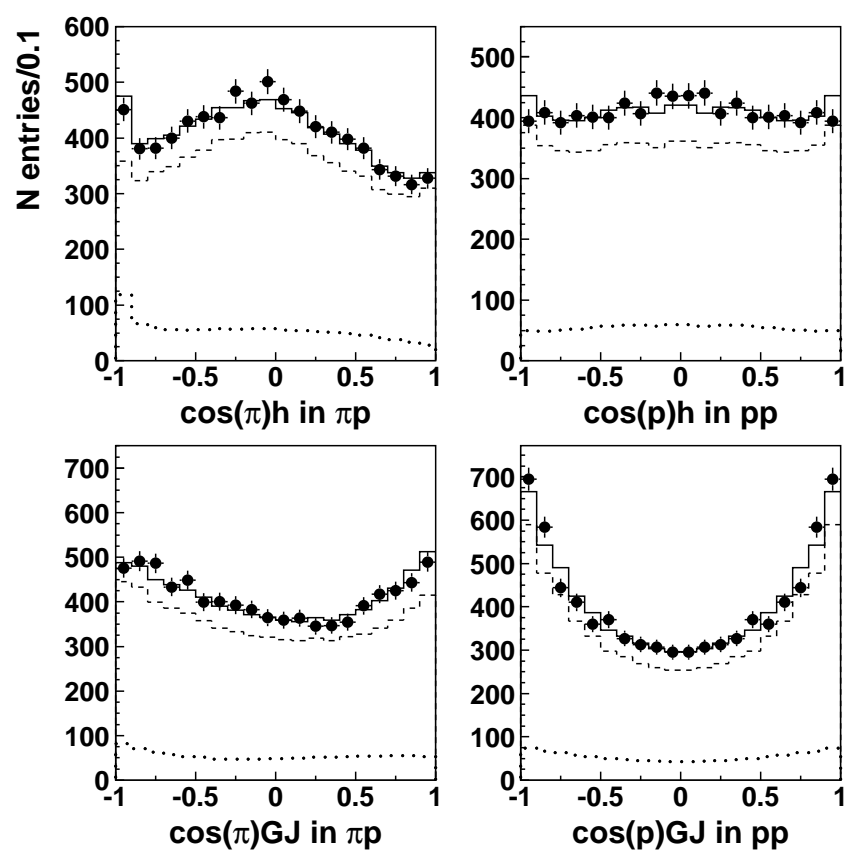

Fig. 4. The angular distribution of the final pion and proton in the helicity and Godfrey-Jackson system for the total interval of neutron momentum. The full histograms are the result of the partial wave analysis, the dashed histograms are the contributions from isovector amplitudes and dotted histograms are contributions from isoscalar amplitudes.

For the $\Delta(1232)$, we use a relativistic Breit-Wigner formula with mass and width fixed from the PDG values. The Roper state was parameterized in agreement with Breit-Wigner couplings found in the analysis 11. Let us note that the present analysis is not sensitive to the exact parametrization of the Roper resonance: only the low energy tail of this state can influence the data.

For the description of the final $p p$ interaction we use a modified scattering length approximation formula:

$$
A_{2 b}^{\beta}\left(s_{i}\right)=\frac{\sqrt{s_{i}}}{1-\frac{1}{2} r^{\beta} q^{2} a_{p p}^{\beta}+i q a_{p p}^{\beta} q^{2 L} / F\left(q, r^{\beta}, L\right)},
$$

where multiindex $\beta$ denotes possible combinations of a kinematical channel $i$ and quantum numbers $S_{2}, L_{2}$ and $J_{2}, a_{p p}^{\beta}$ is a $p p$-scattering length and $r^{\beta}$ is the effective range of the $p p$ system. The $F(q, r, L)$ is the Blatt-Weisskopf form factor (it is equal to 1 for $L=0$ and the explicit form for other partial waves can be found, for example in [8]) and $q$ is a relative momentum in the $p p$-system:

$$
q=\frac{\sqrt{s_{i}-4 m_{p}^{2}}}{2}
$$

For the S-wave partial wave this formula corresponds exactly to the scattering length approximation suggested in [12,13. To check a possible additional energy dependence we also introduced a more complicated parameterization of the numerator in Eq. (8) by substituting:

$$
\sqrt{s_{i}} \rightarrow \sqrt{s_{i}}+b^{\beta} s_{i}
$$

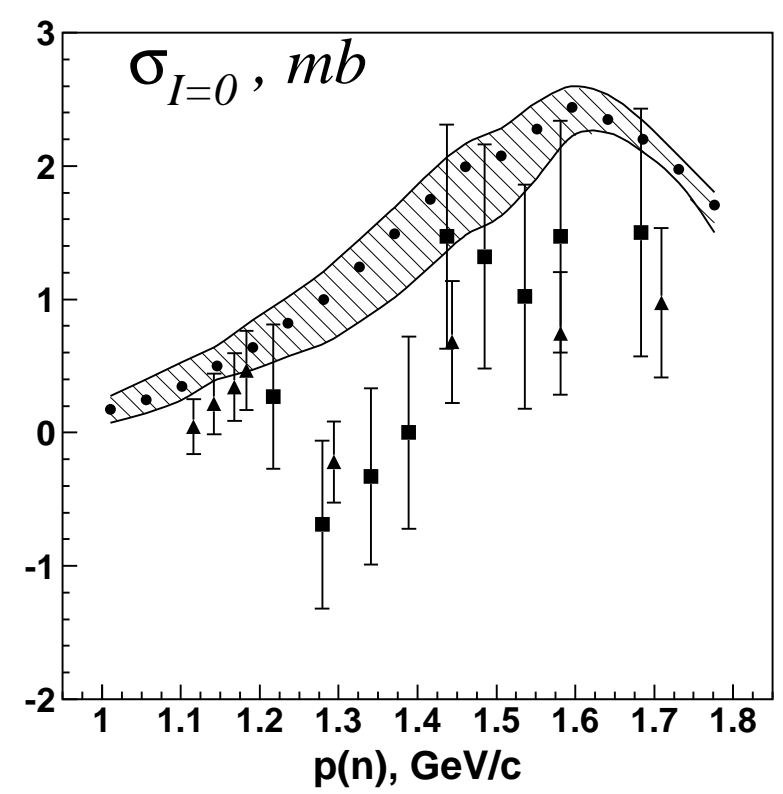

Fig. 5. The isoscalar total cross section. The band represents the systematical errors obtained from the large number of fits. The experimental points are taken from [15] (squares) and 17] (triangles).

where $b^{\beta}$ are fit parameters. However we found that this freedom does not improve the description but leads to large correlations between these parameters and parameters of the transition amplitude $A_{t r}^{\alpha}(s)$.

\section{The results and discussion}

We minimized the log-likelihood value fitting simultaneously the present data taken in the whole range of the neutron momenta and data obtained earlier on $p p \rightarrow p p \pi^{0}$ 14, 15] measured at nine energies covering the same energy interval as the $n p$ data. To fix the low energy region one set of high statistics data taken by the Tübingen group [16] on $p p \rightarrow p p \pi^{0}$ was also introduced to the fit. The $p p$ collision events are produced solely from the isovector channel $(I=1)$ and therefore the contribution of isovector states to the $n p \rightarrow p p \pi^{-}$reaction (which differs only by a common Clebsch-Gordan coefficient) is strongly constrained. This appreciably increases the accuracy for the extraction of the isoscalar contribution from the data.

The results of the partial wave analysis are shown in Figs. 2 3 by solid curves. Contributions of the isovector channel are given by dashed curves and contributions from isoscalar channel as dotted curves. It is seen that the fit reproduces well the asymmetry of the pion angular distribution in the overall c.m.s. (see Fig. 2). Moreover, the angular distribution of isoscalar channel changes notably with energy demonstrating contributions from different partial waves.

The largest contributions from isovector initial states to the pion production process stem from the ${ }^{3} P_{2},{ }^{3} P_{1}$ waves (see Table 1). At large neutron momenta the ${ }^{3} F_{2}$ 
Table 1. Largest contributions of initial partial waves $\left({ }^{2 S+1} L_{J}\right)$ to the reaction $n p \rightarrow p p \pi^{-}$. The central values are given for the best fit and errors are extracted from the large number of different solutions.

\begin{tabular}{cc|cc}
\hline \hline \multicolumn{2}{c}{$I=1$} & \multicolumn{2}{c}{$I=0$} \\
\hline${ }^{3} P_{0}$ & $3.7 \pm 1.3 \%$ & ${ }^{3} S_{1}$ & $0.9 \pm 0.2 \%$ \\
${ }^{3} P_{1}$ & $21.6 \pm 3.0 \%$ & ${ }^{1} P_{1}$ & $10.9 \pm 1.2 \%$ \\
${ }^{3} P_{2}$ & $46.1 \pm 5.0 \%$ & ${ }^{3} D_{1}$ & $1.8 \pm 0.3 \%$ \\
${ }^{1} D_{2}$ & $4.7 \pm 1.5 \%$ & & \\
${ }^{3} F_{2}$ & $10.3 \pm 2.5 \%$ & & \\
\hline \hline
\end{tabular}

contribution is also significant. The ${ }^{1} S_{0}$ wave gives the negligible contribution. The ${ }^{3} P_{2}$ state decays almost equally into the $\Delta(1232) p, N(1440) P_{11} p$ and $\left({ }^{3} P_{2}\right)_{p p} \pi$ states. The ${ }^{3} P_{1}$ initial state decays also almost equally into $\Delta(1232) p$ and $\left({ }^{3} P_{2}\right)_{p p} \pi$, while ${ }^{3} F_{2}$ decays dominantly into the $\Delta(1232) p$ and $N(1440) P_{11} p$ final states. The dominant isoscalar state ${ }^{1} P_{1}$ decays dominantly into $\left({ }^{3} P_{1}\right)_{p p} \pi$ and $N(1440) P_{11} p$, while ${ }^{3} S_{1}$ and ${ }^{3} D_{1}$ states decay almost totally into the $\left({ }^{1} S_{0}\right)_{p p} \pi$ channel.

The quality of the partial wave analysis is also demonstrated in Fig. 4 Here, angular distributions of the pion and final proton are compared to the fit in the helicity and Godfrey-Jackson frames for the total momentum range of the incident neutron. The helicity frame is a rest frame of the two final particles with the angle calculated between one of constituent particles and a spectator particle. This frame is most suitable for the investigation of processes when two initial particles form a system (e.g. resonance) which decays into a final two-body system and a spectator. The Godfrey-Jackson frame is defined as rest frame of two final particles, however here the angle is calculated between one of constituent particles and the beam. This system is mostly suitable for the study of two particle systems in the final state produced by $t$-channel exchange. We would like to mention that the description of these distributions at the same momentum intervals as given in Figs. 213 has the same quality as a total distribution.

The partial wave analysis defines the relative contribution of the isovector and isoscalar channels to the total cross section. The isovector total cross section was measured experimentally [14,15] which provides the possibility to define the energy dependence of the total cross section for the $n p \rightarrow p p \pi^{-}$reaction.

A comparison of the isoscalar cross section found in the partial wave analysis with results from other experiments extracted as:

$$
\sigma(I=0)=3\left[2 \sigma\left(n p \rightarrow p p \pi^{-}\right)-\sigma\left(p p \rightarrow p p \pi^{0}\right)\right],
$$

is shown in Fig. 5 , The points inside the band correspond to our best solution. The band itself shows the systematical errors obtained from a large set of solutions with different parameterizations of partial wave amplitudes. It is seen that our result is systematically exceeds the previous calculations.

The isoscalar cross section increases smoothly from 1.1 $\mathrm{GeV} / \mathrm{c}$ up to $1.6 \mathrm{GeV} / \mathrm{c}$ where it reaches a value of $2.5 \mathrm{mb}$

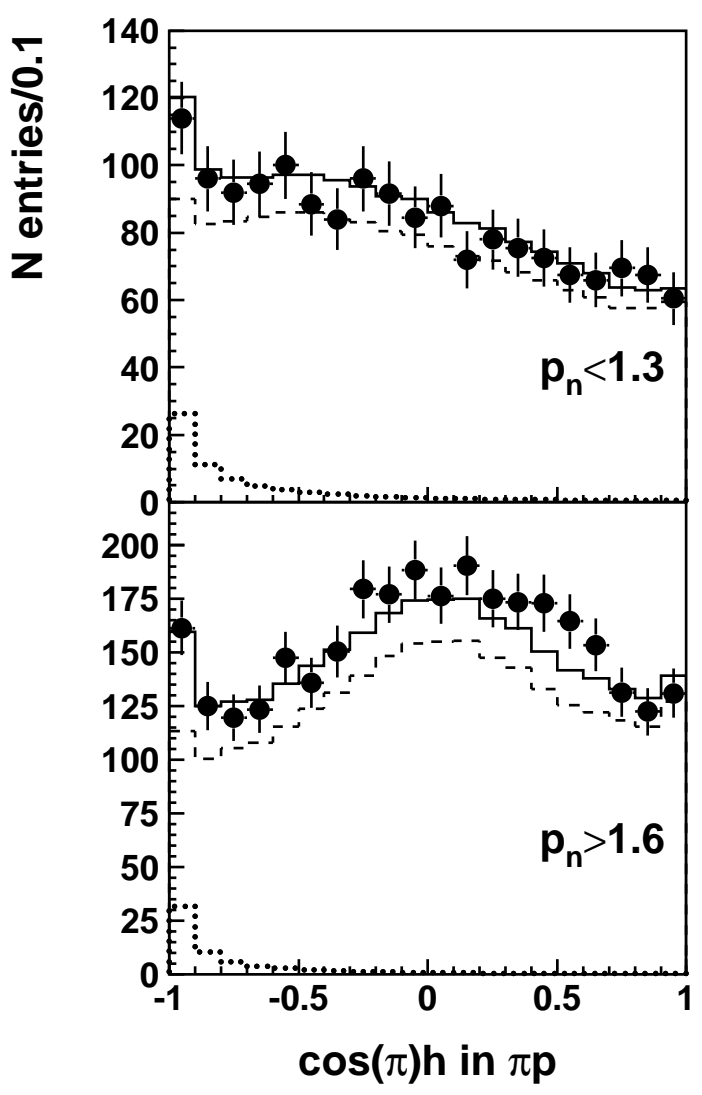

Fig. 6. The angular distribution of the final pion in the helicity system for two intervals of incident neutron momentum (in $\mathrm{GeV} / \mathrm{c}$ ). The full histograms (close to the data points) are the result of the partial wave analysis, the dashed histograms show the contributions from isovector channel and dotted histograms the contributions from isoscalar channel decaying into the $\left({ }^{1} S_{0}\right)_{p p} \pi$ final state.

and then it drops sharply down to $1.5 \mathrm{mb}$. It is possible that this drop is connected with the opening of double pion production channels where isoscalar $n p$ states could decay, for example, into two $\Delta$ states.

The isoscalar initial channel provides a very good tool for a determination of the scattering length of the final $p p$ system in the pion production reactions. In the initial fits we fixed scattering length and effective range of the ${ }^{1} S_{0}$ final $p p$ state to $a_{p p}=-7.83 \mathrm{fm}$ and $r=2.8 \mathrm{fm}$ [18. For other partial waves the effective ranges were also fixed to $r=2.8 \mathrm{fm}$ while parameters $a_{p p}^{\beta}$ were fitted freely.

An appreciable fraction of the isoscalar channel decays into pion and the $p p$ system in the final ${ }^{1} S_{0}$ state. The ${ }^{1} S_{0}$ state produces a sharp peak in the pion backward angle in the helicity frame. This angle corresponds to the lowest $p p$ invariant mass and due to kinematical conditions (suppression of other partial waves), this peak is well observed in the data. The pion angular distributions in the helicity frame for two intervals of the incident neutron momentum is shown in Fig. 6. It is seen that the isoscalar channel produces a dominant effect at small and large momenta of incident neutron. If the effective range is fixed 
at $2.8 \mathrm{fm}$, we found the scattering length to be equal to $a_{p p}=-7.5 \pm 0.3 \mathrm{fm}$.

\section{Conclusions}

We performed a detailed study of the $n p \rightarrow p p \pi^{-}$reaction. The data were analyzed in a maximum likelihood partial wave analysis. The partial wave analysis provides a solid determination of weak isoscalar amplitudes from their interference with strong isovector amplitudes. The dominant decay of the initial isoscalar states ${ }^{3} S_{1}$ and ${ }^{3} D_{1}$ into the $\left({ }^{1} S_{0}\right)_{p p} \pi$ final state allows us to obtain a good determination of the $p p$ scattering length in the final state of pion production reactions.

\section{Acknowledgements}

We would like to express our gratitude to the bubble chamber staff as well as to the laboratory assistants, which toiled at the film scanning and measuring. We are grateful E. Klempt for useful discussions and reading the paper. Part of the work was supported by a FFE grant of the Research Center Jülich and by the Deutsche Forschungsgemeinschaft within the Sonderforschungsbereich SFB/TR16.

\section{References}

1. J.G. Rushbrooke et al., Nuovo Cim. 33, 1509 (1964).

2. D.C. Brunt et al., Phys. Rev. 187, 1856 (1969).

3. L.G. Dakhno et al., Phys. Lett. B 114, 409 (1982).

4. T. Tsuboyama et al., Nucl. Phys. A 486, 669 (1988).

5. M. Kleinschmidt et al., Z. Phys. A 298, 253 (1980).

6. W. Thomas et al., Phys. Rev. D 24, 1736 (1981).

7. V.I. Medvedev et al., Phys. Atom. Nucl. 9 (in press) (2009).

8. A.V. Anisovich et al., Eur. Phys. J. A 24, 111 (2005).

9. A.V. Anisovich et al., Eur. Phys. J. A 30, 427 (2006).

10. A.V. Anisovich et al., Eur. Phys. J. A 34, 129 (2007).

11. A.V. Sarantsev et al., Phys. Lett. B 659, 94 (2008).

12. K.M. Watson, Phys. Rev. 88, 1163 (1952).

13. A.B. Migdal, JETP 1, 2 (1955).

14. V.P. Andreev et al., Phys. Rev. C 50, 15 (1994).

15. V.V. Sarantsev et al., Eur. Phys. J. A 21, 303 (2004).

16. S. Abd El-Samad et al., Eur. Phys. J. A 30, 443 (2006).

17. G. Rappenecker et al., Nucl.Phys. A 590, 763 (1995).

18. Martin L. Gursky and Leon Heller, Phys.Rev. 136, B1693 (1964). 